\title{
A Spitzer Warm Mission Ultra-Wide Survey As A Target Finder For The James Webb Space Telescope
}

\author{
Jonathan P. Gardner*, Xiaohui Fan ${ }^{\dagger}$, Gillian Wilson** and Massimo \\ Stiavelli* \\ ${ }^{*}$ NASA Goddard Space Flight Center, Code 665, Greenbelt, MD 20771, USA \\ ${ }^{\dagger}$ Steward Observatory, University of Arizona, 933 N. Cherry Ave, Tucson, AZ 85721, USA \\ ${ }^{* *}$ Spitzer Science Center, Caltech MS 220-6, Pasadena, CA 91125, USA \\ ${ }^{\ddagger}$ Space Telescope Science Institute, 3700 San Martin Dr, Baltimore, MD 21218, USA
}

\begin{abstract}
The James Webb Space Telescope (JWST) is the successor to the Hubble and Spitzer Space Telescopes. It has a broad scientific mission which includes spectroscopic studies of the epoch of reionization through observations of $z>8$ quasars. The Spitzer warm mission provides a unique opportunity to conduct an infrared survey of several hundred square degrees to a depth of several micro-Janskys, capable of finding quasars out to $z=10$. Deep JWST continuum spectroscopy of these quasars will establish the epoch and history of the Universe through detection of the GunnPeterson trough and/or Lyman- $\alpha$ damping wings. The statistics and luminosity function of high-z quasars will reveal the early history of accretion in the most extreme systems, providing insights in the role of black holes in galaxy evolution. Data obtained from an ultra-wide warm Spitzer survey will also be useful for other science, including studies of high-redshift galaxy clusters.
\end{abstract}

Keywords: Spitzer Space Telescope, infrared astronomical observations, extragalactic objects, origin and formation of the universe

PACS: 95.85.Hp, 98.62.Py, 98.62.Ai, 98.80.Bp

\section{INTRODUCTION}

The James Webb Space Telescope (JWST; Gardner et al. [1]) will be a large infraredoptimized space telescope launched in 2013. It is designed to address four scientific goals: The End of the Dark Ages: First Light and Reionization; The Assembly of Galaxies; The Birth of Stars and Proto-planetary Systems; and Planetary Systems and the Origins of Life. The science goals require high sensitivity in both imaging and spectroscopy in the near and mid infrared. JWST studies of the first galaxies, and their evolution until the present day, will be done with deep and deep-wide imaging and spectroscopic surveys. Studies of stellar and planetary systems will primarily be done with targets in star-forming regions that have already been identified.

JWST studies of reionization, however, will require the identification of bright sources that can be used for absorption spectroscopy. These sources would be quasars at $z>8$, with brightnesses of a few micro-Jy. It is unlikely that JWST can find suitable targets by itself, as they are expected to be no more common than one per $10 \mathrm{~s}$ to $100 \mathrm{~s}$ of square degrees; JWST will not be able to survey an area this large. The JWST imaging field of view is comparable to Hubble's at $10 \operatorname{arcmin}^{2}$, and while the observing and survey efficiency is higher than Hubble's, it will not be orders of magnitude better. Spitzer, with

\footnotetext{
CP943, The Science Opportunities for the Warm Spitzer Mission Workshop, edited by L. J. Storrie-Lombardi and N. A. Silbermann (C) 2007 American Institute of Physics 978-0-7354-0457-1/07/\$23.00
} 
a $25 \operatorname{arcmin}^{2}$ imaging field of view, and comparatively rapid re-pointing capability, is a much more efficient surveyor than either Hubble or JWST; the largest extra-galactic Spitzer survey is $50 \mathrm{deg}^{2}$, while the largest Hubble survey is $2 \mathrm{deg}^{2}$. Finding $\mathrm{z}>8$ quasars will require a survey of hundreds of square degrees to micro-Jy depths in the infrared, with optical support for identification. The Spitzer warm mission will provide a unique opportunity to conduct such a survey.

\section{JWST STUDIES OF REIONIZATION}

Neutral Hydrogen atoms first formed in the early universe when primordial protons and electrons joined together with the release of the cosmic microwave background radiation, about 380,000 years after the Big Bang. About 400 million years later, the first stars and galaxies formed out of the very-low metallicity primordial gas. The first stars were very massive, 30 to $100 \mathrm{M}_{\odot}$ or more, and lived only a few million years. They produced large amounts of ultraviolet (UV) radiation. The UV radiation reionized their neighborhoods, blowing bubbles in the surrounding neutral gas. As more galaxies formed, these bubbles expanded and become more numerous, joined by bubbles of ionized gas surrounding the build-up of black holes in the centers of the galaxies. Eventually, the bubbles of ionized gas joined together, and the universe was reionized.

Soon after the first light sources appear, both high-mass stars and accretion onto black holes become viable sources of ionizing radiation. We do not know which was primarily responsible for reionizing hydrogen in the surrounding intergalactic medium (IGM). Active galactic nuclei (AGN) produce a highly energetic synchrotron power spectrum, and would reionize helium as well as hydrogen. Because observational evidence reveals that helium was reionized at a much later time, hydrogen was probably reionized by starlight at earlier epochs. However, it is possible that helium recombined after being reionized for the first time together with hydrogen. A second Helium reionization would occur during the epoch when quasar activity peaks. Recently, a combination of observations by the WMAP of the cosmic microwave background polarization (Kogut et al. [2], Page et al. [3], Spergel et al. [4]) with spectra of $z>6$ quasars found by the Sloan Digital Sky Survey (SDSS) (Fan et al. $[5,6]$ ) has revealed the possibility that reionization was an extended process. In some models, the completion of the reionization epoch that is seen at $\mathrm{z}=6$ was proceeded by an earlier partial reionization beginning in the first light epoch (Cen [7, 8]). Although the observations allow for other possibilities, there is evidence that the reionization history of the universe was complex (e.g., Gnedin [9]).

The most direct observational evidence of re-ionization is the detection of a GunnPeterson trough (Gunn and Peterson [10]) in the spectrum of high redshift quasars. Neutral hydrogen clouds along the line of sight (the Lyman- $\alpha$ forest) produce increasing absorption as the redshift increases. At $\mathrm{z} \sim 5$, some signal is detected shortward of the Lyman- $\alpha$ line, suggesting that the universe is fully ionized at $z=5$ and that re-ionization was completed at still higher redshifts.

Fan et al. [11, 12, 13]) detected high redshift quasars using the Sloan Digital Sky Survey, including some at $\mathrm{z}>6$, showing a drop in continuum flux just shortward of Lyma- $\alpha$ as much as a factor 150 (see Fig. 1). This is evidence that a Gunn-Peterson trough has been detected in these objects (Becker et al. [14], Fan et al. [6]). Other QSOs 


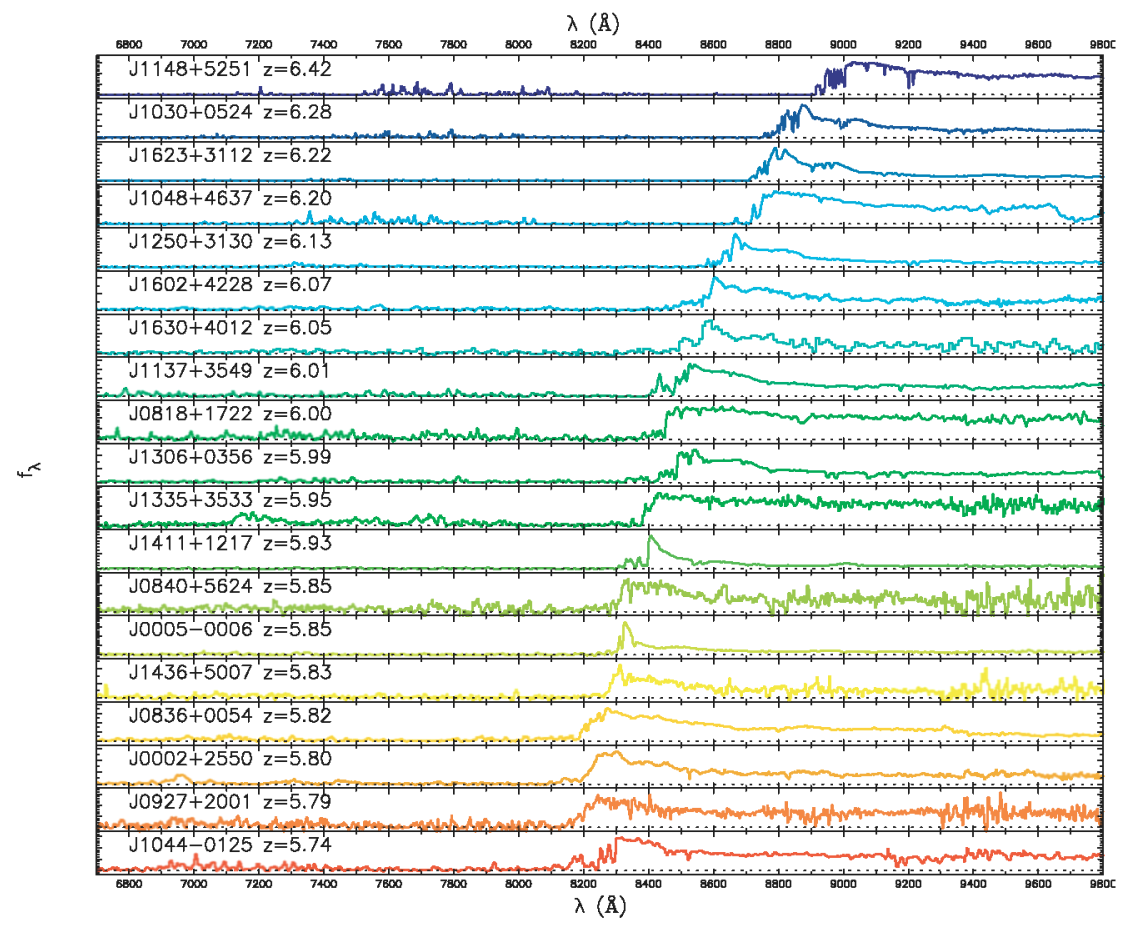

FIGURE 1. Spectrum of 19 SDSS quasars at $5.74<z<6.42$. The absence of flux in the region shortward of the Lyman- $\alpha$ line is a possible indication of a Gunn-Peterson trough in the highest redshift quasars, indicating that the fraction of neutral hydrogen has increased substantially over this redshift range, and that the universe is approaching the epoch of complete reionization at $\mathrm{z} \sim 6$ (From Fan et al. [15]).

at slightly lower redshift show a much smaller continuum drop. Variation in the QSO properties indicates that the reionization did not occur abruptly at the same time throughout the universe. Haiman and Holder [16] argue for an extended 'percolation' period of reionization. We cannot conclude that the reionization epoch has been determined on the basis of these few objects, particularly since even a very modest local neutral hydrogen column density could produce the observed Gunn-Peterson troughs. However, these detections open up the possibility that re-ionization was completed by the relatively low redshift of $\mathrm{z} \sim 6$.

The correlations between the cosmic microwave background temperature and polarization, as measured by WMAP, support a much earlier reionization of hydrogen, giving $\mathrm{Z}_{\text {reion }}=10.9_{-2.3}^{+2.7}$, under the assumption of a single epoch of full reionization (Fig. 2; Spergel et al. [4]). This may be an indication that hydrogen at least partially recombined after the first epoch of reionization, only to be reionized again at a lower redshift. In contrast to the epoch of hydrogen reionization, the epoch of helium reionization has been firmly identified at $\mathrm{z} \sim 3$ through the detection of a Gunn-Peterson trough in quasar spectra (Jakobsen et al. [17], Davidsen et al. [18], Heap et al. [19]).

It is possible to compute the minimum surface brightness required to reionize the 


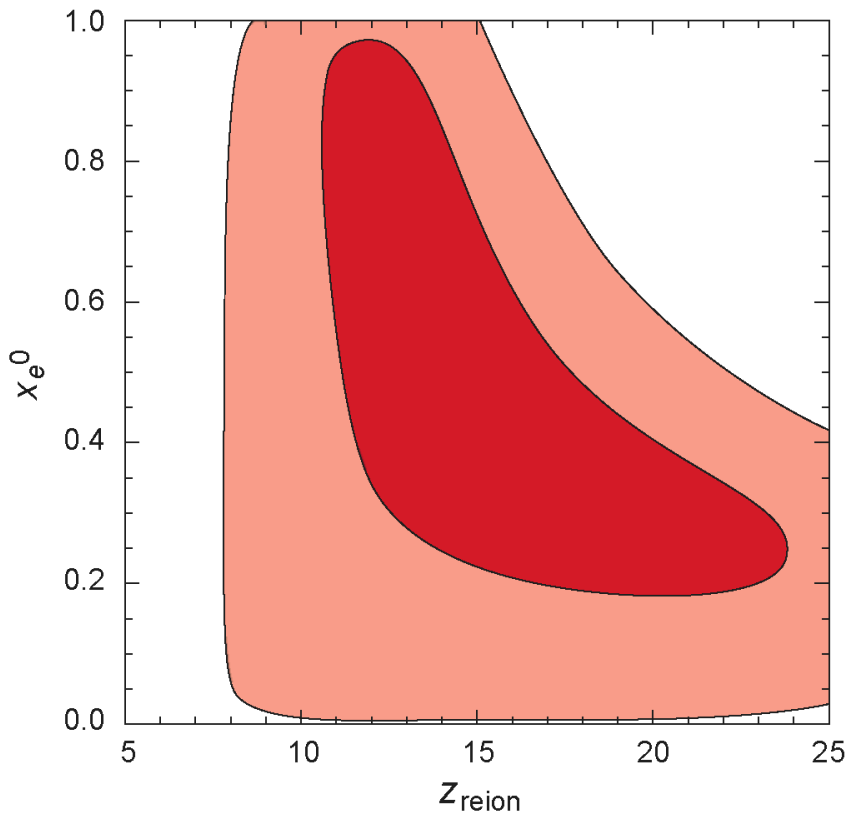

FIGURE 2. WMAP constraints on the reionization history. The plot shows the $68 \%$ and $95 \%$ joint two-dimensional marginalized confidence level contours for a model in which the Universe is partially reionized with an ionization fraction $\mathrm{x}_{e}^{0}$ at $z_{\text {reion }}$, and then fully reionized at $\mathrm{z}=7$. The WMAP data are inconsistent with a single epoch of reionization at $\mathrm{z} \sim 6$, and argue for a complex reionization history. (From Spergel et al. [4]).

universe, under the assumptions that the universe was reionized by hot population III stars, and that all UV photons can escape the system. This minimum surface brightness of ionizing sources at $\mathrm{z}>6$ is about $\mathrm{AB}=29 \mathrm{mag} \operatorname{arcmin}^{-2}$ in a redshifted $\lambda=1400 \AA$ band (Stiavelli et al. [20]), when counted as the typical ionizing flux seen per unit area. For a luminosity function similar to that of $\mathrm{z}=3$ Lyman break galaxies and with $\mathrm{M}^{*}$ not fainter than $-15 \mathrm{mag}$, this implies a few sources per square arcmin with $\mathrm{AB}=31$ or brighter.

Even though one often refers to the epoch of reionization as if it were a sudden transition, the time elapsed between the epochs when $10 \%$ and $90 \%$ of hydrogen was reionized can last a significant fraction of the age of the universe. The WMAP detection of a significant Compton opacity is evidence of either an extended reionization process, or of two distinct reionization epochs (Cen [7, 8], Haiman and Holder [16], Holder et al. [21], Stiavelli et al. [20]). Regardless of the specifics of the reionization process, inhomogeneities along the line of sight may create dispersion in optical depth shortwards of Lyman- $\alpha$. Moreover, only a very low residual fraction of neutral hydrogen is needed to produce a Gunn-Peterson trough in the spectra of high redshift quasars. In addition, 
the opacity near Lyman- $\alpha$ would be modified in the neighborhood of ionizing sources (Miralda-Escudé and Rees [22]), in analogy to the proximity effect in QSOs (Møller and Kjaergaard [23]).

While models differ significantly in the details of how the reionization was started by these various possible first light populations at $15<\mathrm{z}<25$, they all converge to produce roughly the same cosmic star-formation history of population II stars in the mini halos of dwarf galaxies at $6<\mathrm{z}<10$. This is simply the consequence of the need to fit the nearly complete Gunn-Peterson troughs now seen in the spectra of at least four SDSS quasars in the range $6.05<\mathrm{z}<6.43$ (Fan et al. [12]). While these indicate non-zero flux shortward of $0.8 \mu \mathrm{m}$, there is essentially zero flux longwards of $0.810 \mu \mathrm{m}$. Hence, there was significant $\mathrm{HI}$ in front of these quasars at $\mathrm{z}>5.7$, although the HI-fraction at $\mathrm{z}=6$ was still very small (of order $10^{-4}$ to $10^{-5}$ ).

In most models, the conclusion of this reionization epoch is modeled by dwarf galaxies producing an increasing number of population II stars at $6<z<11$. Most models are rather similar in their predictions of the cosmic star formation history at $6<\mathrm{z}<8$, in order to match the SDSS Gunn-Peterson troughs seen at $z=6$. For example, the Cen [7] models predict an increase in the cosmic star-formation history of a full factor of 10 over $16>z>11$ and another factor of 10 increase over $11>z>6$. In other words, most of the population II stars that we see today were born in dwarf galaxies, but most were not born until about $\mathrm{z}=8$ (consistent with the oldest ages of population II measured today of $12.8 \mathrm{Gyr}$ ), and it was likely the high-mass end of those population II stars that completed the epoch of reionization by $z=6$. In WMAP cosmology (Spergel et al. [24]) there was only $300 \mathrm{Myr}$ between at $6<\mathrm{z}<8$ and another $170 \mathrm{Myr}$ at $8<\mathrm{z}<10$, so the stellar population that was formed in those galaxies, and whose O, B and A stars helped complete the reionization of the universe by $\mathrm{z}=6$, is still visible as the low-mass population II stars seen today.

\section{JWST OBSERVATIONS}

The epoch of reionization is revealed through signatures in the Lyman- $\alpha$ forest: a black Lyman- $\alpha$ Gunn-Peterson trough, islands in the Lyman- $\alpha$ forest, and appearance of a Lyman- $\alpha$ damping wing. In addition to these techniques, the epoch of reionization can be identified as the redshift at which there is fast evolution of the properties of Lyman- $\alpha$ emitters. However, a sharp transition in the Lyman- $\alpha$ luminosity function can be suppressed if, for instance, a relatively long reionization onset is coupled to a smooth increase in metal content. Sources at higher redshifts will have increasingly more absorbed Lyman- $\alpha$ but also increasingly stronger intrinsic equivalent widths because of the lower metallicity. It is easy to build models where the two effects cancel out. Alternative methods, not sensitive to this limitation, are the study of the evolution of the ratio between Lyman- $\alpha$ and Balmer lines.

JWST will make deep spectroscopic observations of $\mathrm{z}>8$ quasars to study the Lyman$\alpha$ forest. High signal-to-noise, $\mathrm{R} \sim 1000$, near-infrared spectra will reveal the presence of a Gunn-Peterson trough or of a Lyman- $\alpha$ damping wing. High $\mathrm{S} / \mathrm{N}$ is needed to discriminate between optical depths $\tau$ of a few and $\tau \gg 10$. A damping wing should be present for a few million years, before the ionizing radiation is sufficient to create a 
large Strömgren sphere around each ionizing source. Failure to detect a damping wing does not necessarily imply that the universe is ionized. $\mathrm{R} \sim 100$ spectra will be able to determine the presence of a Lyman- $\beta$ 'island'. This is relevant if reionization occurs relatively abruptly. In this case, objects at redshifts between the redshift of reionization, $\mathrm{z}_{\text {reion }}$, and $\mathrm{z}=\left(\lambda_{\alpha} / \lambda_{\beta}\right)\left(1+\mathrm{z}_{\text {reion }}\right)-1$, will show an island of normal, finite, forest absorption between the Lyman- $\alpha$ and the Lyman- $\beta$ forests. Here, $\lambda_{\alpha}$ and $\lambda_{\beta}$ are the rest frame wavelengths of Lyman- $\alpha$ and Lyman- $\beta$, respectively.

If there are indeed two distinct reionization epochs, the (possibly partial) recombination following the first reionization may be detectable in continuum spectra of high redshift objects as an absorption signature in the region shortward of Lyman- $\alpha$.

\section{HIGH REDSHIFT QUASARS}

Discoveries of luminous quasars at $\mathrm{z} \gtrsim 6$ (Fan et al. [5, 12, 13], Fan, Carilli, and Keating [25], Mahabal et al. [26], Cool et al. [27], McGreer et al. [28]) show that active, massive black holes of order a billion solar masses existed a few hundred million years after the first star formation in the Universe. Their absorption spectra reveal the rapid evolution of the IGM at the end of reionization.

\subsection{The Quasar Luminosity Function: Black Hole Growth at $\mathrm{z}>6$}

Evolution of the density and bright-end luminosity function of quasars have been well-studied using the SDSS sample at $0<\mathrm{z}<6$ (Fan et al. [11, 13], Richards et al. [29], Hopkins et al. [30]). At $z>3$, the density declines exponentially towards high redshift: for quasars at $\mathrm{M}_{B}<-27$, the density at $\mathrm{z} \sim 6$ is a factor of about 40 lower than where it peaks at $\mathrm{z} \sim 2.5$ (see Fig. 3). Meanwhile, the slope of quasar luminosity also appears to be a strong function of redshift: while it is relatively flat $\Phi(\mathrm{L}) \propto \mathrm{L}^{-2.5}$ at z $\sim 4$, it appears to have considerably steepened at $\mathrm{z} \sim 5$ to $\mathrm{z} \sim 6$, with $\Phi(\mathrm{L}) \propto \mathrm{L}^{-3.1}$, suggesting a large population of faint quasars at high-redshift (see Fig. 4). These faint quasars could also be a significant contributor of the reionization photon budget.

The existence of such objects is surprise. The SDSS $\mathrm{z} \sim 6$ quasars are among the most luminous quasars at any redshift; their apparent magnitudes are about 19 even at $\mathrm{z}>6$ ! Their black hole masses are in the range $10^{9}$ to $10^{10} \mathrm{M}_{\odot}$, and they are likely to reside in dark matter halos of $10^{13} \mathrm{M}_{\odot}$, comparable to the most massive black holes and galaxies found locally. The black hole masses are close to the theoretical maximum allowed by Eddington-limited accretion for black hole growth. It is a challenge to understand how the universe managed to assemble these systems so fast and so efficiently; this rapid growth places one of the strongest constraints on the theory of supermassive black holes.

The evolution of the high-redshift quasar luminosity function provides direct constraints on the accretion mode of the highest redshift quasars. The standard supermassive black hole model assumes that it grows from a stellar mass seed black hole through radiatively efficient, Eddington-limited accretion and black hole-black hole mergers. Assuming a radiative efficiency of 0.1 for black hole accretion, the Salpeter time, defined as the e-folding time for black hole growth in Eddington-limited accretion, is about $4 \times$ 


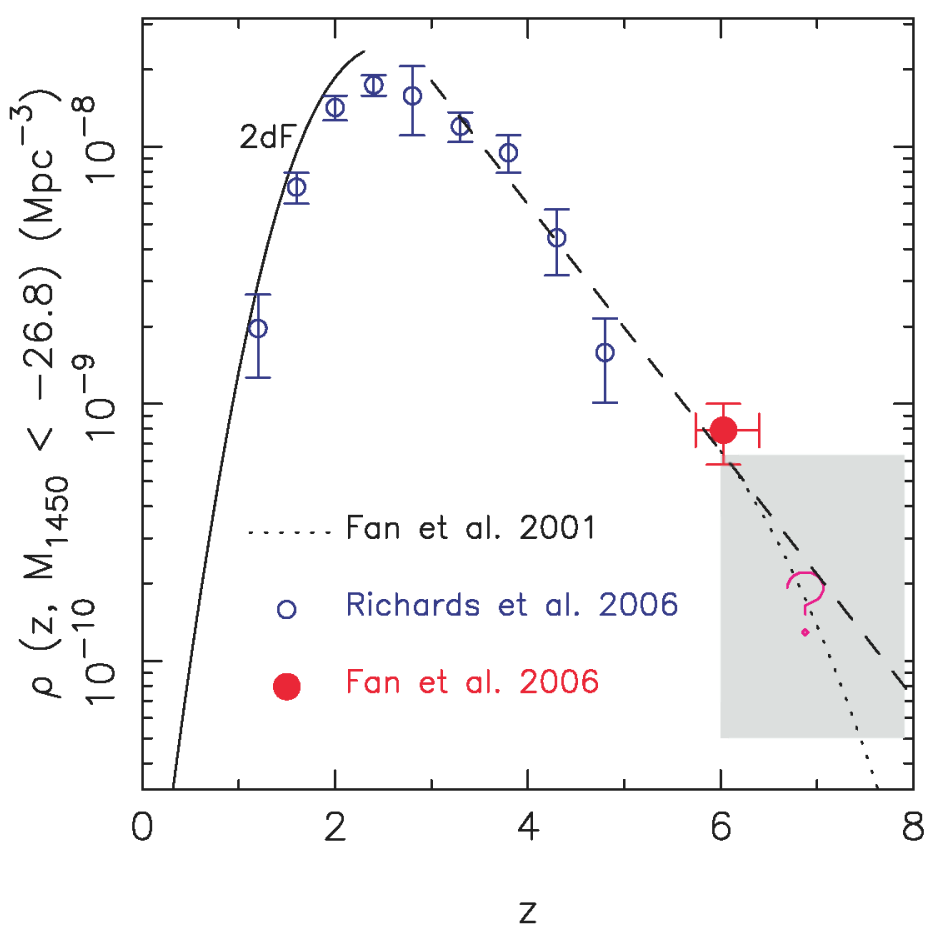

FIGURE 3. Evolution of the quasar number density. The quasar number density at $\mathrm{z} \sim 6$ is a factor of 40 times lower than the peak at $\mathrm{z} \sim 2$. It is unknown whether the slope continues to higher redshift, or whether the evolution is even steeper.

$10^{7} \mathrm{yr}$. Even if the first supermassive star formed as early as $\mathrm{z} \sim 30$, there would only be about 15 Salpeter times available for black hole growth until $\mathrm{z} \sim 7$. If quasars with $10^{9} \mathrm{M}_{\odot}$ existed at $\mathrm{z} \sim 7$, even non-stop Eddington accretion requires the existence of seed black holes with $\mathrm{M}>300 \mathrm{M}_{\odot}$, probably collapsed from massive Pop III stars (e.g., Heger et al. [31], Abel et al. [32], Tumlinson et al. [33]).

A number of negative feedback effects would further slow down black hole growth. Using semi-analytic modeling, Volonteri and Rees [34] recently modeled black hole growth through accretion and mergers, including feedback effects such as gravitational recoil and growth timescale changes due to black hole spin-up. They conclude that unless super-Eddington accretion or direct formation of intermediate-mass black holes with $10^{3}$ to $10^{6} \mathrm{M}_{\odot}$ is important, high-redshift black holes have to be limited to moderate spin (with low radiative efficiency) and the formation needed to be highly selective at early epochs. They predict that there will be a rapid decline of quasar density and a rapid steepening of the quasar $\mathrm{LF}$ at $\mathrm{z}>6$, as the growth of the most massive black holes is limited in that case by the number of e-folding times available. The steepening LF at $\mathrm{z} \sim 6$ discovered in the SDSS, if confirmed towards higher redshift, would strongly suggest that the Salpeter timescale constraint is becoming the limiting factor for black 


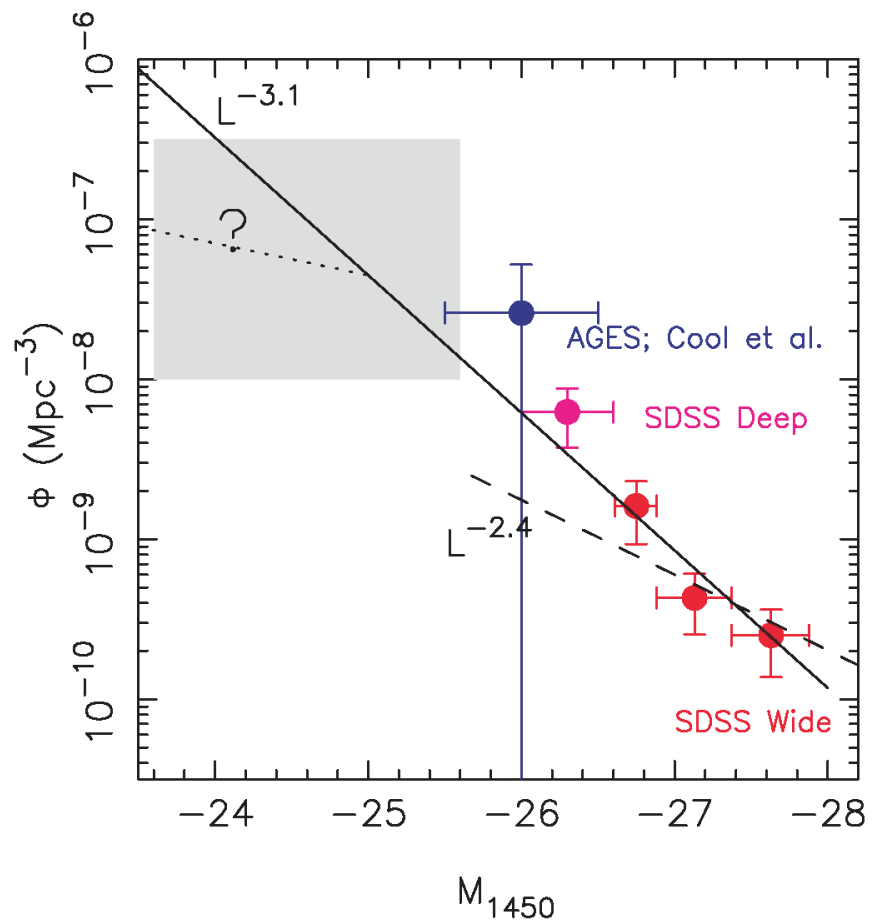

FIGURE 4. Quasar luminosity function at $\mathrm{z} \sim 6$. The luminosity function of quasars at $\mathrm{z} \sim 6$ detected by the SDSS and the Spitzer AGES survey shows a steeper slope $\left(\mathrm{L}^{-3.1}\right)$ than at lower redshift. A break is expected at faint magnitudes, but is currently undetected.

hole growth. New quasar samples will enable the determination of the quasar LF down to $\mathrm{M}_{1450} \sim-23.5$, possibly reaching the expected break of the $\mathrm{LF}$ at $\mathrm{z}>6$.

Discovery of any extremely luminous quasar at $\mathrm{z}>7$ or beyond will present a major challenge to the theory of black hole formation. To overcome the timescale constraint, a number of models (e.g. Madau [35], Begelman et al. [36], King and Pringle [37]) suggest rapid early black hole growth either through super-Eddington accretion, or direct formation of intermediate-mass black holes. Recently, Li et al. [38] used hydrodynamic simulations that include both hierarchical galaxy formation and black hole feedback effects to study the growth of $z>6$ quasars. They show that in a large simulated volume, for Eddington-limited accretion, only the most biased peaks in the most extreme galaxy formation environment could have reproduced observed properties of luminous quasars at $\mathrm{z} \sim 6$ towards a small fraction of selected viewing angles. Detailed comparison between the simulated and observed quasar LF at $6<\mathrm{z}<8$ (Fig. $3 \& 4$ ) will test whether the earliest supermassive black holes can still grow by Eddington accretion, or alternative accretion modes are truly needed for their rapid growth.

The discovery of such quasars at $z>7$ would answer several key questions: Will their properties be very different from those at lower redshift? Will they pose a serious 
challenge to our current models of Eddington-limited black hole growth and hierarchical galaxy formation?

\subsection{Evolution of Quasar Properties at High Redshift}

One of the somewhat surprising results from the studies of SDSS quasars is the similarity in the quasar spectral energy distributions (SEDs) between high and low$\mathrm{z}$ quasars. This is especially evident in UV and X-ray observations, which trace the emission from the broad line region and accretion disk. Fan et al. [13] presented the average spectrum of a sample of $\mathrm{z} \sim 6$ quasars in the $U V$; it is virtually identical to the standard quasar template constructed from low-redshift quasars, in terms of both emission line strengths and continuum shape. Using emission line ratios, they showed that quasar broad emission line regions have roughly solar or even higher metallicities at $\mathrm{z} \sim 6$ (e.g., Pentericci et al. [39], Freudling et al. [40], Dietrich et al. [41], Venkatesan et al. [42]). This lack of evolution demonstrates that the emission line region and accretion disk can form on a short timescale and are decoupled from the cosmological environment.

However, there is now some evidence for the possible evolution in properties of the AGN dust torus and host galaxy that are further away from the central engine. Most lowredshift dust is generated in AGB star envelopes; at $\mathrm{z}>6$, there is not yet enough time for intermediate mass stars to evolve. This timescale constraint suggests possible evolution in the dust origin and properties. Spitzer observations reveal two $\mathrm{z} \sim 6$ quasars that do not show any sign of hot dust emission in mid-IR wavelengths, while no such quasar has ever been found at lower redshift (Jiang et al. [43]). Some SDSS z $\sim 6$ quasars also exhibit dust extinction inconsistent with any standard extinction curve, but can be fit by extinction generated from the much smaller grains that are produced in short-lived Type II supernova envelopes (Maiolino et al. [44]). It will be highly exciting if the emission line properties of quasars also show strong evolution when we reach $\mathrm{z}>7$.

Luminous high-redshift quasars are likely to be located in the densest environments in the early Universe. However, direct imaging of the stellar light of host galaxies is extremely difficult with current technology. At observed far-IR to $\mathrm{mm}$ wavelengths, radio-quiet quasars are dominated by the reprocessed radiation from cool dust in the host galaxy. Roughly $40 \%$ of the radio-quiet quasars are detected at the $1 \mathrm{mJy}$ level at $1 \mathrm{~mm}$ wavelength (e.g. Carilli et al. [45], Priddey et al. [46], Wang et al. [47]). Their FIR luminosity $\left(\sim 10^{13} \mathrm{~L}_{\odot}\right)$ implies a dust mass of order $10^{8} \mathrm{M}_{\odot}$. If the dust heating came from starburst activity, that would suggest star formation rates as high as $10^{2}$ to $10^{3} \mathrm{M}_{\odot} \mathrm{yr}^{-1}$. Molecular gas emission from these starforming host galaxies provides the only method so far to constrain their dark matter halo masses at $\mathrm{z} \sim 6$. Walter et al. [48] obtained high-resolution VLA observations of the $\mathrm{CO}$ in the $\mathrm{z}=6.42$ quasar SDSS J1148+5251. The gas has a line-width of $280 \mathrm{~km} / \mathrm{s}$, with the size of the CO emitting region $\sim 2 \mathrm{kpc}$. This is the first time a high-redshift quasar host is resolved both spatially and kinematically. Assuming the gas is bound, the estimated dynamical mass is about $10^{10} \mathrm{M}_{\odot}$ within the $2 \mathrm{kpc}$ radius. The total bulge mass is about one order of magnitude lower than one would expect from the local black hole mass - velocity 
dispersion relation. This result, as well as $\mathrm{CO}$ measurements in other quasars at $\mathrm{z} \sim 4$ (Shields et al. [49]), indicate that luminous quasars at high redshift do not live in a fully developed galaxies with massive central bulges. Does this suggest that the black hole formed before galaxy assembly? Pushing such studies to higher redshift will require JWST and ALMA.

\subsection{Spitzer Warm Mission Survey for Quasars at z $>7$}

Given the uncertainties in black hole accretion and feedback models and dust obscurations, it is currently still difficult to make accurate theoretical calculations of the density of quasars at $z>7$. However, Volonteri and Rees [34] predict a relatively steep luminosity function at $\mathrm{z} \sim 6$, consistent with the new SDSS observations. They also find relatively smooth evolution in the black hole mass function at high redshift. Meanwhile, $\mathrm{Li}$ et al. [38] show that the progenitors of the $\mathrm{z} \sim 6$ quasars are roughly one and two orders of magnitude fainter in bolometric luminosity at $\mathrm{z} \sim 8$ and $\mathrm{z} \sim 10$, respectively, although the energy output of AGN light in the rest-frame optical and UV is highly uncertain. These calculations suggest that a simple extrapolation of the SDSS quasar luminosity function from $\mathrm{z} \sim 6$ is possible.

A major consideration in survey design is the issue of depths vs. area. A steep quasar luminosity function suggests a higher efficiency with deeper coverage; however, the quasar luminosity function will have an unknown break in the slope at fainter luminosities. At $\mathrm{z} \sim 6$, this break occurs at $\mathrm{M}_{B}>-24$. The same luminosity corresponds to $\mathrm{f}_{3.6}=1.5 \mu \mathrm{Jy}$ at $\mathrm{z}=8$, and $\mathrm{f}_{3.6}=1 \mu \mathrm{Jy}$ at $\mathrm{z}=10$. We estimate the number of quasars to be detected with a Spitzer warm mission in two surveys. A shallow survey of 500 $\operatorname{deg}^{2}$ down to $\sim 3 \mu \mathrm{Jy}$ (SWIRE depth) will detect about 130 quasars at $\mathrm{z}>6,22$ quasars at $z>8$ and 0.8 quasars at $z>10$. Because the steepness of the luminosity function approximately cancels the effects of $\sqrt{t}$, a deeper survey of $50 \mathrm{deg}^{2}$ observed to $\sim 1$ $\mu \mathrm{Jy}$ will give approximately the same number of quasars: about 120 quasars at $\mathrm{z}>6$, 20 quasars at $z>8$ and 0.7 quasars at $z>10$. Although these two straw-man surveys would find about the same number of quasars, the quasars in the deeper survey would be fainter. As JWST requires high signal-to-noise continuum spectra for its reionization studies, the wider survey would be better suited.

While a Spitzer survey can detect these high-z quasars, candidate selection and identification observations are not trivial. These quasars have $\mathrm{J}_{A B} \sim 23$ to 24 . Even with VISTA, there is no ground-based JHK survey reaching this depth in such wide area. So the candidate selection will likely rely on combining Spitzer and deep optical (i, z, and Y) data. For example, the planned PANSTARRS medium deep survey reaches $\mathrm{z}_{A B}=$ 24.7 and $\mathrm{Y}_{A B}=23.9$ over $1200 \mathrm{deg}^{2}$.

The main contaminants are cool dwarfs and dusty, red galaxies at intermediate redshift. Cool dwarfs are much brighter in the 3.6 and 4.5 micron bands, and have much redder [3.6]-[4.5] colors. Most of the low-redshift galaxies can also be separated out by combining optical and Spitzer colors (e.g. Stern et al. [50]). More detailed modeling is required to refine the color selection for dusty galaxies and whether they will present a major challenge to the color selection. Once the candidate list has been reduced to a few 
hundred sources, then ground-based near-infrared follow-up of individual candidates will be possible. Spectroscopic confirmation may require JWST.

\section{OTHER SCIENCE FROM AN ULTRA-WIDE SPITZER SURVEY}

A wide-area survey is ideal for finding rare objects. The power of Spitzer lies in its ability to rapidly survey large areas of the sky to depths of a few $\mu \mathrm{Jy}$ in the infrared, reaching normal galaxies at redshifts of $1<\mathrm{z}<3$. The most massive objects in the Universe are clusters of galaxies, and their existence at high redshift places strong constraints on both cosmology and galaxy evolution. Crucial stages in cluster galaxy evolution, where vigorously star-forming proto-cluster regions at $2<\mathrm{z}<6$ (Kurk et al. [51], Steidel et al. [52]) transform into the quiescent early-type population seen at $\mathrm{z} \sim 1$ (Blakeslee et al. [53], Holden et al. [54]) appear to occur in the hard-to-access redshift range $1<\mathrm{z}<2$, the current 'cluster desert,' where clusters have been difficult to find and identify. Recent results from Spitzer (Stanford et al. [55], Wilson et al. [56], Brodwin et al. [57]) have shown that it is possible to detect clusters at $\mathrm{z}>1$ using IRAC's 3.6 and/or 4.5 micron channels in combination with optical data. The largest of the IRAC cluster surveys is being carried out by the SpARCS collaboration in the 50 square degree SWIRE fields (Muzzin et al. [58], Wilson et al. [59]) and uses an infrared adaptation of the twofilter Cluster Red-Sequence technique (Gladders and Yee [60], Gilbank [61], Gladders and Yee [62]). The SpARCS survey is now about two-thirds complete, and using a combination of z'-band and IRAC 3.6 micron imaging, is on track to discover a total of about 200 new clusters at $1<z<2$ by the survey's end in late 2007. Several of these clusters are awaiting spectroscopic confirmation and are candidates for the most massive distant objects yet discovered. In the coming years, the number density of these new massive clusters in the $\mathrm{z}>1$ redshift range has the potential to place important independent constraints on cosmological parameters (Gladders et al. [63]), and in particular, on the equation of state of the dark energy.

In addition to studies of high-z quasars and clusters, an ultra-wide survey would extend all of the science results from the short-wavelength SWIRE survey data with a factor of 10 greater statistics. Results would include luminosity functions, galaxy-galaxy correlation functions, weak lensing dark matter tomography and low-mass field stars.

\section{CONCLUSION}

High-redshift quasars are needed as background sources for JWST studies of reionization, and are interesting in their own right. A $500 \mathrm{deg}^{2}$ survey to the SWIRE depth offers the best chance for finding bright quasars out to $\mathrm{z} \sim 10$.

Deep JWST continuum spectroscopy of bright $\mathrm{z}>8$ quasars will establish the epoch and history of reionization of the Universe through detection of the Gunn-Peterson trough and/or Lyman- $\alpha$ damping wings. The statistics and luminosity function of high-z quasars will reveal the early history of accretion in the most extreme systems, providing insights in the role of black holes in galaxy evolution. 
The data obtained from this survey would also be useful for a wide variety of other science, including constraints on cosmology and galaxy evolution from the detection of $\mathrm{z}>1$ galaxy clusters.

\section{REFERENCES}

1. Gardner, J. P., et al. 2006, Space Science Reviews, 123, 485

2. Kogut, A., et al. 2003, ApJS, 148, 161

3. Page, L., et al. 2007, ApJS, 170, 335

4. Spergel, D. N., et al. 2006, ApJS, 170, 377

5. Fan, X. et al., 2001 b, AJ 122, 2833

6. Fan, X. et al., 2002, AJ, 123, 1247

7. Cen, R. 2003a, ApJ, 591, 12

8. Cen, R. 2003b, ApJL, 591, L5

9. Gnedin, N. Y. 2004, ApJ, 610, 9

10. Gunn, J. E., and Peterson, B. A. 1965, ApJ, 142, 1633

11. Fan, X. et al., 2001a, AJ, 121, 54

12. Fan, X. et al., 2003, AJ, 125, 1649

13. Fan, X. et al., 2004, AJ, 128, 515

14. Becker, R.H., et al. 2001, AJ, 122, 2850

15. Fan, X. et al., 2006, AJ, 131, 1203

16. Haiman, Z., and Holder, G. P. 2003, ApJ, 595, 1

17. Jakobsen, P., et al. 1994, Nature, 370, 35

18. Davidsen, A. F., Kriss, G. A., and Wei, Z. 1996, Nature, 380, 47

19. Heap, S.R., et al. 2000, ApJ, 534, 69

20. Stiavelli, M., Fall, S. M., and Panagia, N., 2004, ApJ, 600, 508

21. Holder, G. P., Haiman, Z., Kaplinghat, M., and Knox, L. 2003 ApJ, 595, 13

22. Miralda-Escudé, J., and Rees, M. J. 1994, MNRAS, 266, 343

23. Møller, P., and Kjaergaard, P., 1992, A\&A, 258, 234

24. Spergel, D. N., et al. 2003, ApJS, 148, 175

25. Fan, X., Carilli, C. L., and Keating, B. 2006, ARA\&A, 44, 415

26. Mahabal, A., Stern, D., Bogosavljevic, M., Djorgovski, S. G., and Thompson, D., 2005, ApJ, 634, L9

27. Cool, R. J., et al., 2006, AJ, 132, 823

28. McGreer, I. D., Becker, R. H., Helfand, D. J., and White, R. L., 2006, ApJ, 652, 157

29. Richards, G. T., et al., 2006, AJ, 131, 2766

30. Hopkins, P. F., et al., 2007, ApJ, 654, 731

31. Heger, A., Fryer, C. L., Woosley, S. E., Langer, N., and Hartmann, D. H., 2003, ApJ, 591, 288

32. Abel, T., Bryan, G. L., and Norman, M. L., 2002, Science, 295, 93

33. Tumlinson, J., Venkatesan, A., and Shull, J. M., 2004, ApJ, 612, 602

34. Volonteri, M., and Rees, M. J., 2006, ApJ, 650, 669

35. Madau, P., 2005, Growing Black Holes: Accretion in a Cosmological Context, 3

36. Begelman, M. C., Volonteri, M., and Rees, M. J., 2006, MNRAS, 370, 289

37. King, A. R., and Pringle, J. E., 2006, MNRAS, 373, 90

38. Li, Y., et al., 2007, ApJ, 665, 187

39. Pentericci, L., et al., 2002, AJ, 123, 2151

40. Freudling, W., Corbin, M. R., and Korista, K. T., 2003, ApJL, 587, L67

41. Dietrich, M., Hamann, F., Appenzeller, I., and Vestergaard, M., 2003, ApJ, 596, 817

42. Venkatesan, A., Schneider, R., and Ferrara, A., 2004, MNRAS, 349, L43

43. Jiang, L., et al., 2006, AJ, 132, 2127

44. Maiolino, R., Schneider, R., Oliva, E., Bianchi, S., Ferrara, A., Mannucci, F., Pedani, M., and Roca Sogorb, M., 2004, Nature, 431, 533

45. Carilli, C. L., et al., 2001, ApJ, 555, 625

46. Priddey, R. S., Isaak, K. G., McMahon, R. G., Robson, E. I., and Pearson, C. P., 2003, MNRAS, 344, 47. Wang, R., et al. 2007, AJ, 134, 617 
48. Walter, F., Carilli, C., Bertoldi, F., Menten, K., Cox, P., Lo, K. Y., Fan, X., and Strauss, M. A., 2004, ApJ, 615, L17

49. Shields, G. A., Menezes, K. L., Massart, C. A., and Vanden Bout, P., 2006, ApJ, 641, 683

50. Stern, D., et al. 2005, ApJL, 631, 163

51. Kurk, J.D., et al.2004, A\&A, 428, 817

52. Steidel, C.C., et al.2005, ApJ, 626, 44

53. Blakeslee, J.P. et al.2003, ApJL, 596, 143

54. Holden, B. et al., 2004, AJ, 127, 2484

55. Stanford, S. A. et al, 2005, ApJ, 634L

56. Wilson, G., et al. 2006, in proceedings 'The Spitzer Space Telescope: New View of the Cosmos', ASP Vol. 357, 238

57. Brodwin, M., et al., 2006, 651, 791

58. Muzzin, A., et al., 2006, in proceedings 'The Spitzer Space Telescope: New View of the Cosmos', ASP Vol. 357, 246

59. Wilson, G., et al. 2007, ApJ, 660, 59

60. Gladders, M. D. and Yee, H. K. C., 2000, 120, 2148

61. Gilbank, D. G., 2004, MNRAS, 348, 551

62. Gladders, M. D. and Yee, H. K. C., 2005, 157, 1

63. Gladders, M. D., et al. 2007, ApJ, 655, 128 